\title{
Functional Recovery and Prediction of Prognosis in Incomplete Spinal Cord Injured Persons
}

\author{
Tetsuo Suyama, MD, $\mathrm{PhD}^{1}$, Kuniyasu Takahashi, MD, PhD ${ }^{1)}$, Shigeru Hirabayashi, $\mathrm{MD}, \mathrm{PhD}^{2}$, \\ Yoshie Takahashi, $\mathrm{PT}^{1}$, , Syusuke Kusano, MD ${ }^{3)}$, Masafumi Mizukami, PT $^{3)}$ \\ ${ }^{1)}$ Department of Rehabilitation, Saitama Medical Center, Saitama Medical School, Kamoda- \\ Tsujidoumachi 1981, Kawagoe City, Saitama 350-8550, Japan. TEL +81 492-28-3690 \\ ${ }^{2}$ Department of Orthopaedic Surgery, Saitama Medical Center, Saitama Medical School \\ ${ }^{3}$ Department of Rehabilitation, National Rehabilitation Center for the Disabled
}

\begin{abstract}
Making a functional prognosis for patients with traumatic incomplete spinal cord injuries is extremely important not only for the patients themselves, in attempting to achieve independence in activities of daily living and social rehabilitation, but also for medical co-workers, the family and almost everyone in the society. However, only very few reports deal with this subject, and rehabilitation is often conducted aimlessly. In the present study we tried at our department to summarize the functional prognosis of patients with spinal injuries and set up a preliminary standard for rehabilitation. The present study included 75 patients with Frankel class C, D, E, incomplete spinal injury who underwent rehabilitation training in the past ten years at our department. The level of the injury was the cervical cord in 53 patients and the thoracic or lumbar cord in 22 patients. The average age of the patients was 36.2 years. Seventy of the patients were male and 5 female. The time of the final evaluation after the injury was on the average 11.8 months ( $\mathrm{SD}=5.9$ months). When muscle power of the lower extremities three months after injury reached [4], patients accomplished independent walking 6.8 months after the injury. When muscle power of the lower extremities 6.5 months after injury reached [3-], patients accomplished semi-independent walking with the help of a wheelchair 8.7 months after the injury. Patients with a muscle power of more than [3-] in the upper extremities could walk independently. Among patients with incomplete injury of the cervical cord 64\% gained independence in ADL 8.6 months after the injury, but patients with central paralysis or strong spasticity required assistance.

Key words: Spinal cord injury, Functional recovery, Prediction of prognosis.
\end{abstract}

(This article was submitted Mar. 11, 1999, and was accepted Jun. 10, 1999)

\section{INTRODUCTION}

A prediction of the degree of functional recovery in the future, independence in the activities of daily living (ADL), and the possibilities of occupational rehabilitation are, of course, extremely important not only for patients with traumatic incomplete spinal cord injuries, but also for medical co-workers, patients' families and society.

Prognosis of the functional recovery from incomplete paralysis is particularly difficult.
Rehabilitation is carried out frequently for a short period and then suspended, or on the contrary, continued unnecessarily long, because its goals have not been clearly defined. Treatment and rehabilitation of spinal cord injuries (SCI), however, require a careful approach and the preparation of a rehabilitation program starting immediately after the injury and continuing after discharge from the hospital until social rehabilitation has been achieved.

Motor function disorders in incomplete paralysis resulting from spinal cord injuries comprise a mixture of secondary motor neuron disorders, partly 
Table 1. Classification of incomplete cervical spinal cord injury

\begin{tabular}{lll}
\hline 1. Cross-sectional type & 36 cases & $(68 \%)$ \\
2. Brown-Sequard type & 1 & $(1.8 \%)$ \\
3. Anterior type & 0 & \\
4. Posterior type & 3 & $(5.7 \%)$ \\
5. Central type & 13 & $(24.5 \%)$ \\
\hline \multicolumn{3}{c}{53 cases } \\
\hline
\end{tabular}

The cross-sectional type is the most frequent form, followed by the central type.

due to involvement of cells in the anterior horn of the gray matter within the injured spinal cord, due to hemorrhage or necrosis and pyramidal disorders due to primary motor neuron disturbances. Thus, in our study we observed frequently a loss of motor function, decreased elaborateness, increased tendon reflexes, pathologic reflexes and similar symptoms. However recovery from paralysis ranges from almost complete to a recovery rendered practically useless because of loss of motor power and spasticity, so that a clear definition of the targets is often very difficult.

In the present study we decided that a knowledge of prognostic factors is important to determine the targets clearly, and in a retrospective study we analyzed the degree of recovery of patients with spinal cord injuries and tried to predict their prognosis and then added some considerations regarding the rehabilitation.

\section{MATERIALS}

This study included 554 patients with spinal injuries hospitalized during the 10 years from October 1981 until September 1991 at the National Rehabilitation Center for the Disabled. Among these 339 patients $(61.2 \%)$ were admitted for rehabilitation. This number included 247 patients (72.9\%) with complete, and 92 patients $(27.1 \%)$ with incomplete paralysis. The present study included 75 of the 92 patients $(81.5 \%)$ with incomplete paralysis presenting with Frankel class C, D or E. Frankel class B, where motor function is completely lost, although sensation is retained was for our purposes the same as Frankel type A regarding motion and were therefore excluded.

(1) Classified by the level of the injury 53 patients $(70.7 \%)$ had a lesion in the cervical cord and 22 patients $(29.3 \%)$ in the thoracic or lumbar cord.

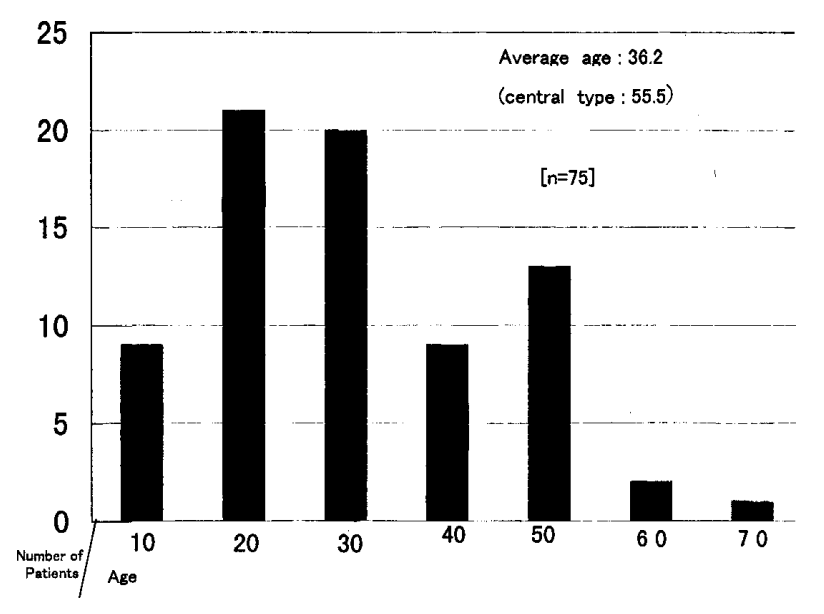

Fig. 1. Age Distribution

This condition is frequent in the 20 s and 30 s, but also among patients in their 50s.

(2) The most frequent type of incomplete paralysis was the transsectional type located in the cervical cord and found in 36 patients. Central paralysis was the next most frequent form found in 13 patients and was followed by the posterior type and the Brown-Sequard type, 1 case each. There were no patients with the anterior form (Table 1).

(3) Lesion was most frequent in patients aged in the 20s and 30s with a little less marked peak in 50s. The average age was 36.2 years. The mean age of patients with the central type, however, was higher, 55.5 years (Fig. 1).

(4) Classified by sex there were 70 men and 5 women.

(5) The most frequent cause for the injury was falls in 26 patients. The next most frequent causes were car accidents (11), sports accidents (10), and motorcycle accidents (9), in that order. This order differs from the order of patients with complete paralysis, where car accidents was the most frequent cause (Fig. 2).

(6) Types of spinal injury were classified as compression fractures in 26 patients $(34 \%)$, dislocation fractures in 16 patients $(21 \%)$ and dislocations in 9 patients (12\%). There were, as many as 25 patients (33\%) without any bone injury.

(7) Treatment.

Treatment was invasive in $72 \%$, and conservative in $28 \%$.

The invasive treatment was an anterior column 


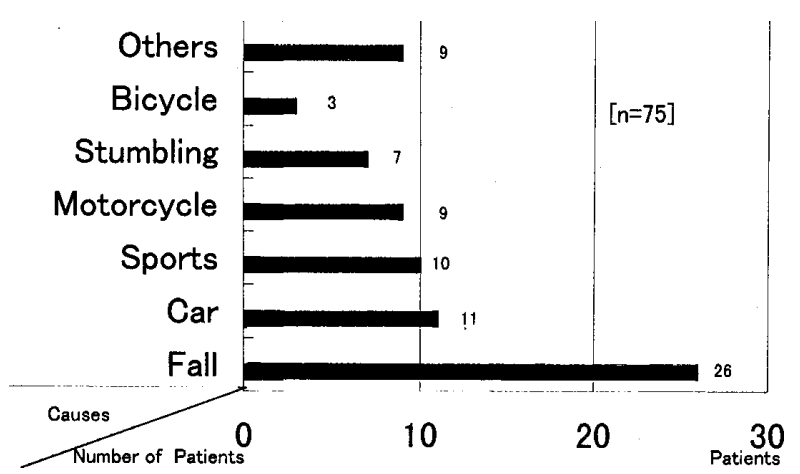

Fig. 2. Causes for the injuries

in 46 patients (cervical vertebra $=33$ cases, thraco-limbar vertebra $=13$ cases), a posterior column in 16 patients (Harrington Rod=3 cases, Luque wire $=1$ Case, pedicular screw $=1$ case) and plastic surgery of the spinal canal in 4 cases (all cervical vertebra).

The duration of immobilization was for conservative treatment on average 1.9 months ( $\mathrm{SD}=0.95$ months) and for invasive therapy an average of 1.6 months ( $\mathrm{SD}=0.96$ months). These values do not differ significantly.

(8) Complications at the time of injury.

The most frequent complication was a treatment loss of consciousness after injury of the cervical cord, and otherwise in 2 patients with injury of the thoracic cord and 3 patients with injury of the lumbar cord, making a total of 16 patients. Fractures of the extremities occurred in 11 patients with injury of the cervical cord and in 3 patients with injury of the lumbar cord, a total of 14 patients. Other complications associated with cervical cord injury were skull, thoracic and pelvic fractures. Thus, many different forms of complications occur with cervical cord injury. The transient loss of consciousness in particular made an accurate evaluation of the paralysis at the time of injury difficult.

(9) The interval between injury and admission to the hospital was on average 6.7 months ( $\mathrm{SD}=1.7$ months). The mean duration of admission in this hospital was 4.5 months ( $\mathrm{SD}=2.9$ months). In this study the final evaluation of the degree of functional recovery was made on average 11.8 months $(\mathrm{SD}=5.9$ months) after the injury.

\section{RESULTS}

I Form of paralysis

We used Frankel's classification ${ }^{4)}$. Frankel class $\mathrm{A}$ is a complete loss of motor and sensory function, Frankel B includes residual sensory function, Frankel $\mathrm{C}$ means residual sensory and motor functions (useless), Frankel D is a (useful) residue of sensory and motor functions and Frankel E indicates recovery of sensory and motor functions, however exaggerated deep tendon reflexs remain here. Most of the patients presenting at the time of injury with Frankel class B or C improved until final evaluation at 11.8 months (approximately 121 months) after the injury to Frankel class D, followed by Frankel group E and C. Patients who were unconscious after the injury or where evaluation was for other reasons not unclear were excluded from the study.

\section{Locomotion}

Following cervical cord injury $42 \%$ of the patients were able to walk semi-independently using a wheelchair, $38 \%$ recovered to walk independently, $12 \%$ required help while in a wheelchair and $8 \%$ became independent sitting in a wheelchair. After thoracic and lumbar cord injury 50\% of the patients gained semi-independent walking ability by using a wheelchair, followed by $27 \%$ who became independent sitting in a wheelchair and $23 \%$ who recovered to independent walking.

When we compared cervical cord trauma with thoracic and lumbar trauma in both groups most patients evidently recovered to manage semi-independent walking. In the former group, however, the number of patients who recovered to be able to walk independently was larger than in the group of patients with central paralysis. Also, in the former group there were some patients presenting with serious tetraplegia, so that they required assistance even when using a wheelchair (Table 2).

III Time of settlement of the form of locomotion

In patients who gained independent walking ability the form of locomotion became fixed after 6.8 months; in patients who accomplished semi-independent walking the form settled after 8.7 months; in those who became independent in a wheelchair after 12.1 months and in those requiring assistance even when using a wheelchair after 18.2 months. 
Table 2. Locomotion ability

\begin{tabular}{lcc}
\hline & $\begin{array}{c}\text { Cervlcal cord injury } \\
\mathrm{n}=53\end{array}$ & $\begin{array}{c}\text { Thoracic and lumbar } \\
\text { cord injury } \mathrm{n}=22\end{array}$ \\
\hline $\begin{array}{l}\text { Semi-independent walking } \\
\quad \text { (simultaneous use of a wheelchair) }\end{array}$ & $42 \%$ & $50 \%$ \\
Independent walking & $38 \%$ & $23 \%$ \\
Independence in a wheelchair & $9 \%$ & $27 \%$ \\
Assisted in a wheelchair & $11 \%$ & \\
\hline
\end{tabular}

Patients with central paralysis gained independent or semi-independent walking ability, but $62 \%$ required assistance for rising.

Table 3. Time of settlement of the form of locomotion

\begin{tabular}{lcl}
\hline \multicolumn{1}{c}{ Locomotion ability } & Time of settlement (after the injury) \\
\hline Independent walking & 6.8 Months & $(\mathrm{SD}=2.06)$ \\
Semi-independent walking & 8.7 Months & $(\mathrm{SD}=1.83)$ \\
Independence in a wheelchair & $12.1 \mathrm{SD}$ & $(\mathrm{SD}=3.45)$ \\
Assisted in a wheelchair & 18.9 Months & $(\mathrm{SD}=5.87)$ \\
\hline
\end{tabular}

In patients who gained early independence the form of locomotion settles correspondingly earlier.

In patients gaining early independence the form of locomotion also settled early. Patients requiring a wheelchair or even assistance while using a wheelchair take a much longer time. The data probably can be interpreted to say that it takes approximately one year to improve from the stage of requiring assistance in a wheelchair to reach independence in a wheelchair (Table 3).

IV Recovery of muscle power in the lower extremities and locomotion ability

We examined the arithmetic mean value of the muscle power of 4 muscles: Gluteus maximus, Quadriceps femoris, Biceps femoris and Tibialis anterior. Plotting the results of the manual muscle test (MMT) on the y-axis and the time passed after injury on the $\mathrm{x}$-axis, the group of patients who attained a mean muscle power of [4] within an average of 3 months after injury was defined as the independent walk group; the group in which a mean muscle power of [3-] was reached within an average of 6.5 months following the injury could be classified as the semi-independent walk group; and those whose muscle power did not exceed $\left[^{+}\right]$could be classified as the wheelchair group. Patients who gained even after an average period of 10 months only a mean muscle power of [2] could be classified as the wheelchair assistance group (Fig. 3).
$\mathrm{V}$ Recovery of muscle power in the upper extremities and locomotion ability after incomplete cervical cord injuries

We examined locomotion ability and the arithmetic mean value of muscle power of the biceps brachii, extensor carpi radialis, triceps and palmar muscles. The group of patients who attained a mean muscle power of $\left[3^{+}\right]$after an average of 5 months following injury was defined as the independent walk group; similarly the group in which a mean muscle power of $\left[3^{+}\right]$was reached after an average of 8 months following the injury was defined as the semi-independent walk group. Those who attained only a value of [3] after an average of 7.6 months could be classified as the wheelchair group. Muscle power of the upper extremities largely influences the ability to walk. Walking requires a muscle power of [3] and patients who reached this level in 5 months could walk independently. Patients remaining below this level of [3] formed the group requiring a wheelchair. The period after the injury also had an important influence on whether independence was achieved or assistance was required (Fig. 4).

IV Degree of independence regarding ADL following cervical cord injury was divided into independent walking, semi-independent walking 


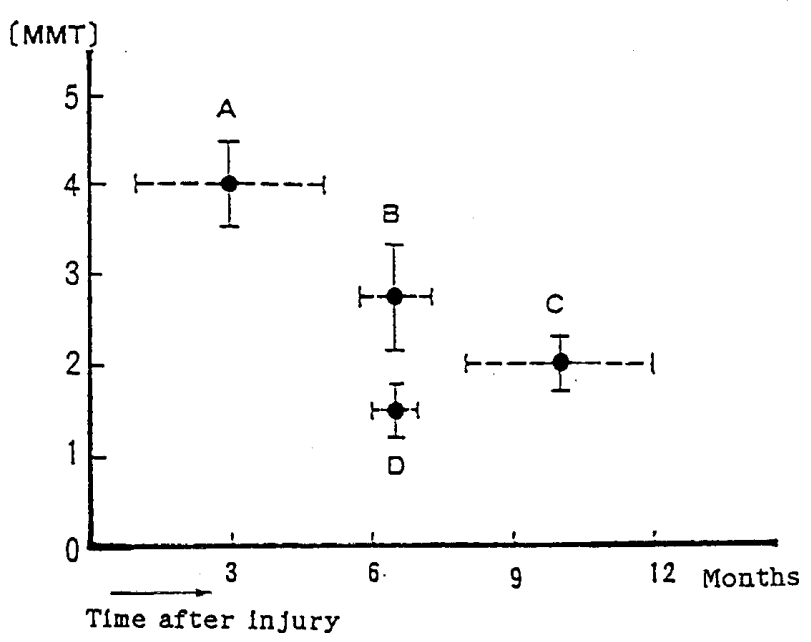

Fig. 3. Recovery of muscle power in the lower extremities and locomotion ability

The muscle power given here is the arithmetic mean of values for the gluteus maximus, quadriceps femoris, biceps of thigh and tibialis anterior muscles.

A: Independent walk group; MMT [4] Within 3 months

B: Semi-independent walk group; MMT $\left[3^{-}\right]$after 6.5 months

C: Wheelchair assistance group; MMT [2] after 10 months

D: Wheelchair independence group; MMT $\left[1^{+}\right]$after 6.5 months

and wheelchair independence. But, in patients with central, partially and assisted ADL occurred both in independent and semi-independent walking patients. The pre-assistance group were considered to be a wheelchair assistance group.

\section{DISCUSSION}

Cheshire $^{2)}$ and Bosch'1), Marar'5) et al. reported classifications for traumatic incomplete spinal cord injuries. These investigators considered various aspects of the paralysis like sensory and motor paralysis, anatomical site of the lesion and similar aspects.

A neurologic improvement can be anticipated when within $24 \mathrm{~h}$ of the injury a sensory sacral sparing occurs. Prediction of the degree to which this recovery will provide functionality for daily living or the possibilities of social rehabilitation is of extreme importance not only for the patients, but also for all related persons.

Disability is classified during the acute phase by

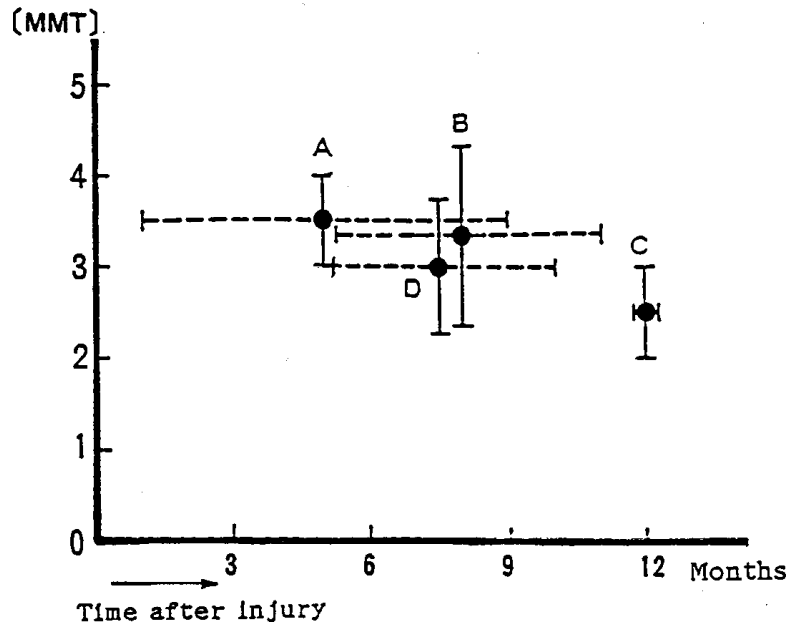

Fig. 4. Recovery of muscle power in the upper extremities and locomotion ability

The muscle power given here is the arithmetic mean of values for the biceps brachii, extensor carpi radialis, triceps and palmar muscles.

A: Independent walk group; MMT $\left[3^{+}\right]$Within 5 months

B: Semi-independent walk group; MMT $\left[3^{+}\right]$after 8 months

C: Wheelchair assistance group; MMT [2] after 10 months

D: Wheelchair independence group; MMT $\left[1^{+}\right]$ after 6.5 months

an evaluation of the neurologic signs and clinical symptoms to make the prognosis of the disability.

Frankel classified neurologic symptoms into five stages and examined functional changes. He reported that 41 of the 62 patients $(66.2 \%)$ with incomplete paralysis at the Stoke Mandeville Hospital recovered to gain practical motor function and $16(25.8 \%)$ recovered almost completely.

Maynard et al.6) reported that 8 of 17 patients $(47 \%)$ in whom sensory functions were observed 72 hours after injury and 27 of 31 patients (87\%) with voluntary motor functions gained walking ability.

Deverat et al. ${ }^{3)}$ used the Yale Scale Score (YSS) to make prognoses for patients with cervical cord injury during the acute phase.

Ohashi et al. ${ }^{7)}$ analyzed the chronic phase in patients with cervical cord injury. According to his investigation patients who regained a muscle power of more than [3] within 2 months after the injury generally could walk independently after an average of 3.3 months, and patients who regained a muscle power of [3 to 4] within 3 to 6 months after 
the injury generally could manage to walk with the use of a stick after an average of 7.1 months.

There are only very few reports dealing with detailed analyses of the chronic phase of incomplete spinal cord injuries. Our study resembles the report of Ohashi et al. in that it shows that independent walking becomes possible when muscle power of the lower extremities reached [4], semi-independent walking at a muscle power of [3-] and at a muscle power of $\left[2^{+}\right]$or less a wheelchair was required. Of course, recovery of the muscle power of the upper extremities largely influences these developments. We did not consider the use of splints or sticks separately. Additionally, no reports deal with muscle power of the upper extremities in relation to rising training, standing or support during walking. The results of the present study indicate that a muscle power in the upper extremities of more than $\left[3^{+}\right]$will permit walking.

Patients with central paralysis can walk and stand at once, but because of the paralysis of fingers and hand do not acquire independent ADL.

Trunk balance and the severity of spasticity also influence whether patients with incomplete spinal injury will be able to perform ADL independently. More accurate information will be necessary for a general evaluation, because functional disorders of upper and lower extremities are not similar, psychological instability and similar complex problems complicate the situation.

\section{CONCLUSION}

(1) We investigated the chronic phase of the recovery of patients with incomplete spinal cord injury who underwent over the past ten years undertook rehabilitation at our department.
(2) The study included 75 patients evaluated to be Frankel class C, D, or E and the duration of the evaluation period was 11.8 months from the injury.

(3) Patients who recovered a muscle power of [4] after 3 months managed to walk independently after 6.8 months.

(4) Patients who recovered a muscle power of [3-] after 6.5 months managed to walk semi-independently after 8.7 months.

(5) Sixty four percent of the patients with incomplete injury to the cervical cord perform ADL independently after 8.6 months, but patients with central paralysis or marked sapsticity required assistance.

\section{REFERENCES}

1) Bosch $A$, et al: Incomplete traumatic quadriplegia: $A$ ten-year review. JAMA 216: 473-478, 1971.

2) Cheshire DJE: A classification of the functional endresults of injury of the cervical spinal cord. Paraplegia 8: 70-73, 1971.

3) Deverat P, et al: Early prognostic factors for walking in spinal cord injuries. Paraplegia 26: 255-261, 1988.

4) Frankel HL, et al: The value of postural reduction in the initial management of closed injuries of the spine with paraplegia and tetraplegia. Paraplegia 7: 179192, 1969.

5) Marar BC: The pattern of neurological damage as an aid to the diagnosis mechanisms in cervical spine injuries. JBJS

6) Maynard, F.M. et al: Neurological prognosis after traumatic quadriplegia. Three-year experience of California regional spinal cord injury care system. J. Neurosurg 50: 611-616, 1979.

7) Ohashi Y, et al: Study on the goal setting for ambulation in patients with incomplete spinal cord injuries. Sogo rehabilitation 6: 605-611, 1982. 